\title{
Intelligent Number Plate Detection System
}

\author{
Madhu Nakerikanti ${ }^{1}$, Y. Chakrapani ${ }^{2}$, Raja Murali Prasad ${ }^{3}$ and R. Hemalatha ${ }^{4}$ \\ ${ }^{1,3}$ Department of ECE, Vardhaman College of Engineering, Hyderabad, Telangana, India \\ ${ }^{2}$ Department of ECE, ACE Engineering College, Hyderabad, India \\ ${ }^{4}$ Department of ECE, Osmania College of Engineering, Hyderabad, Telangana, India
}

\begin{abstract}
In this paper, the automatic detection of the number plate using INTEL EDISON to diminish the time taken by the police to discover the criminal for the violations that are going on utilizing vehicles has described. Open CVEt Numpy are libraries display in python utilized as a part of extraction of the characters and numbers which are available in the number plate of the vehicle. The input to the libraries would be a picture caught utilizing the camera. The intention of this system is to build an application that may help the police in discovering the culprits inside no time. The character models are prepared initially. The characters on the number plate would be of government given characters. We would prepare it i.e., train it with the goal that the characters show in the tag will be distinguished.
\end{abstract}

\section{KEY WORDS: INTEL EDISON, IMAGE SENSOR, YOCTO LINUX, AMAZON WEB SERVICES (AWS), OPEN CV, NUMPY.}

\section{INTRODUCTION}

The fast advancement in people in general transportation framework, has brought out automatic identification of vehicles which has been assuming a critical part in a major part of the applications all through the previous two decades. For illustrations, the identification might use for dealing with the parking facilities, entering of unapproved vehicles into the private zones can be checked, recognition of stolen vehicles, traffic volume can be controlled, ticketing fast vehicles, etc. A standout amongst the best and valuable recognizable proof techniques utilized as a part of numerous applications in visual image processing. Three handling modules are created in the Automatic License Plate Recognition (ALPR) framework; they are, number plate discovery, character division, and character recognition. Among them, the assignment "number plate identification" is most significant stage in the entire ALPR framework. Hues, vertical edges, symmetry, corners, so forth, are the significant highlights utilized for number plate recognition technique.

Biosc Biotech Res Comm P-ISSN: 0974-6455 E-ISSN: 2321-4007
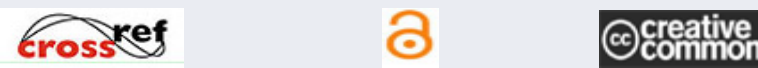

Identifiers and Pagination

Article Information

Year: 2021 Vol: 14 No (5) Special Issue

Received: $17^{\text {th }}$ Mar 2021

Pages: $355-358$

Accepted after revision: $27^{\text {th }}$ May 2021

This is an open access article under Creative

Commons License Attribn 4.0 Intl (CC-BY).

DOI: $h t t p: / / d x . d o i . o r g / 10.21786 / b b r c / 14.5 / 61$
2. Design Of System: In this application a camera for catching the pictures of the vehicles continuously is utilized. Camera is associated with Intel Edison which acts as edge device which implies that it can perform mathematical calculations by utilizing Open CV functions with a foundation support of Numpy Matrix operations and sends the information to cloud. The input to Intel Edison is the picture caught by a camera. Now picture will be processed by the Edison. The processing includes recognition of the number plate of the vehicle. It is the most essential criteria since we need to know whether there is a vehicle or not in that picture. Recognition of number plate will be the hardest activity in this.

2.1 Number Plate Detection: Number plate is an example made out of a few characters which have high characteristic intensities in their background. The key element to recognize the desired number plate is the high contrast part in the image.

\section{System Hardware And Software:}

3.1 Intel Edison: Intel Edison was developed by Intel, It is a computer-on-module, It can be used as a development system for IoT Application devices. Initially the system was declared to be an indistinguishable shape and size from that of a SD card. It Consists a Intel dual-core Quark x86CPU at $400 \mathrm{MHz}$ communicating via Wi-Fi Ct Bluetooth. Then after the CPU was changed to a $22 \mathrm{~nm}$ Silvermont Intel dual-core Atom CPU, and a moment form of Edison was appeared at IDF in September 2014, the size of which greater and thicker than a standard SD card. 
The way of looking at the embedded electronics can be changed using this platform of ultra-small computing. While providing the strong quality of go-to single board computer, every Edison stuff with a gigantic measure of tech goodies into a small bundle. To append a veritable slew of shield-like "blocks" which can stack over each other, a BLE, Wi-Fi, and a 70-pin connector include in the system powered by Intel Atom on Chip Dual-core CPU. The Add-on for Intel Edison can be the base block which permits to append distinctive peripherals like a keyboard, mouse, or thumb drive. Anything which connects to a USB can be presently associated with the Edison which is equipped with a micro AB USB supported by USB OTG and FT231X respectively and we should guarantee that there are no issues in joining outer equipment to Edison.

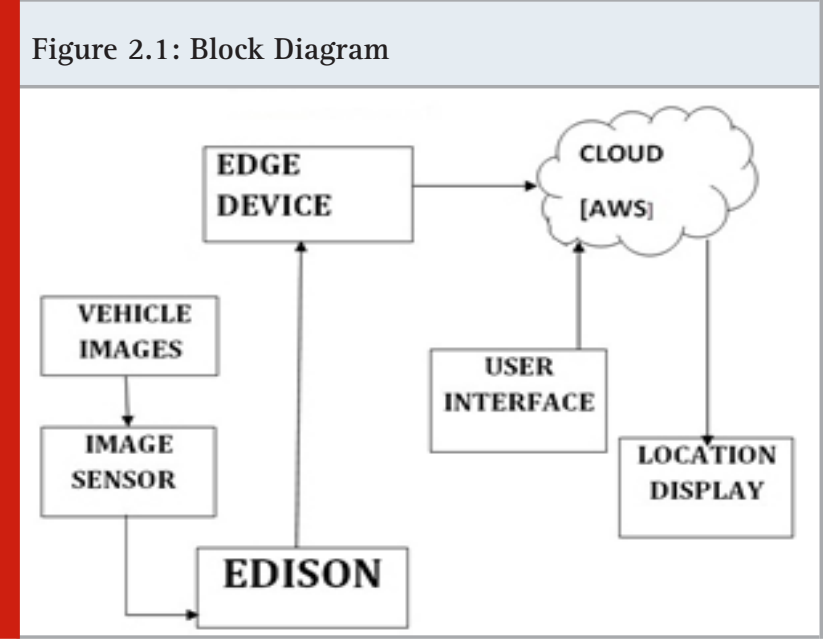

Figure 4.1: Hardware Execution

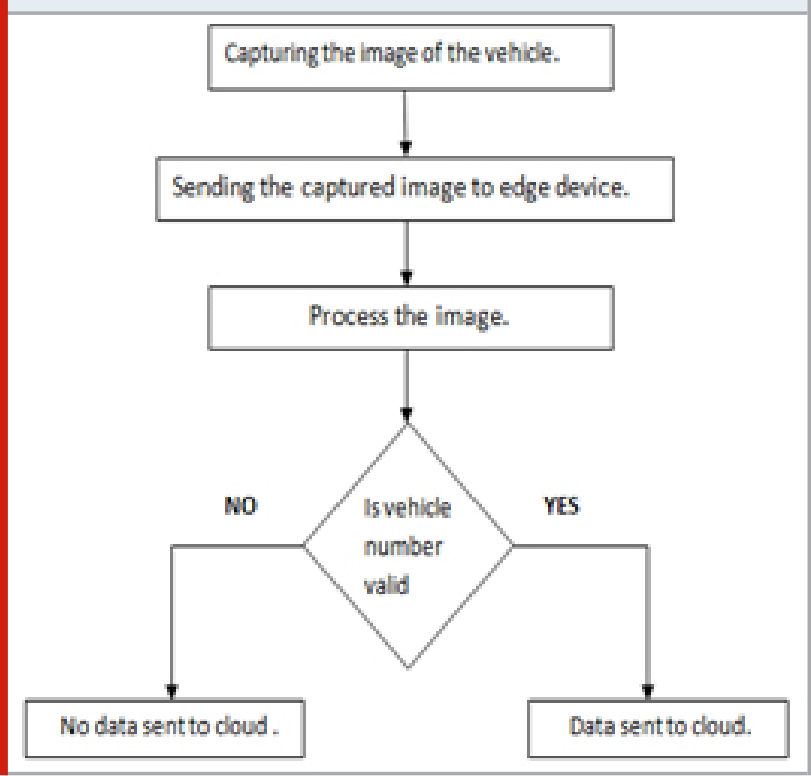

3.2 Image sensor: A webcam could be a camera that streams its image increasingly through or to a computer to a computer network. The video stream can be viewed after it was caught by the computer and it has shared or sent to different systems completely based on that of another frameworks. For example, web and assigned as a connection. If the video stream sent to a remote area, it may be viewed, saved, or sent to others. By using a comparable cable or a USB cable or embedded with PC equipment like laptops, a webcam is connected not like an IP camera which associates based on Wi-Fi or Ethernet.

\section{Figure 4.3: Webpage Execution}

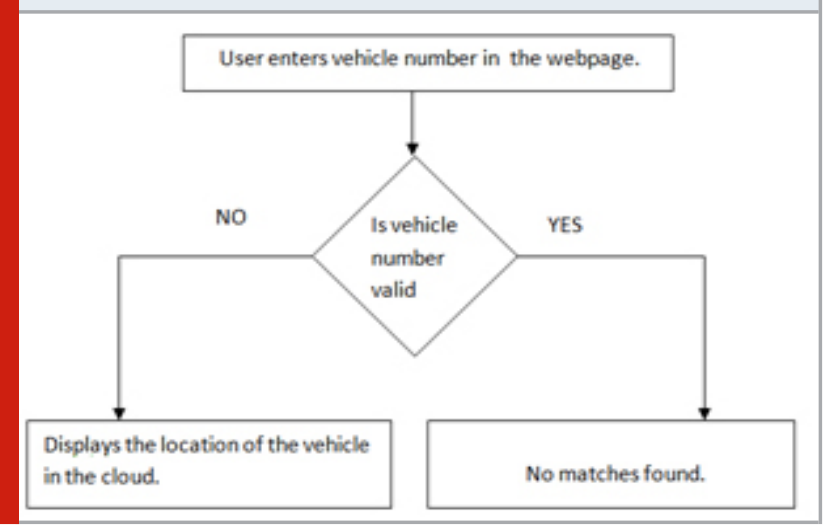

3.3 Yocto Linux: The project of Yocto is a collaborative open source project of Linux Foundation that focuses on delivering devices and procedures that aides in empowering the linux disseminations making for embedded programming. These are free of the inserted hardware's hidden engineering. In 2010, the Linux foundation was announced the project in 2010 in collaborative effort with 22 associations involving Open Embedded and it was propelled in March 2011. The primary focal point of the Yocto Project's is to enhance the product advancement process for embedded Linux distributions. The repeatable and fast advancement of Linux-based implanted frameworks empower the metadata, interoperable tools, and procedures that are given by the Yocto Project, in which each part of the improvement procedure can be tweaked.

3.4 PuTTY: PuTTY is a free terminal and an open-source emulator, system record exchange and serial comfort application. Different types of network protocols have supported such as Raw Socket Association, Telnet, SSH, Rlogin, and SCP. It will consider as the interface using a port. The porting of PuTTY has performed for different alternative frameworks but composed initially for Microsoft Windows. For a few Unix-like phases, official ports can be accessible with work-in advance ports to MacOS and Classic mac OS. Informal ports have superimposed into the stages. For example, Windows Mobile, Symbian, and Windows Phone.

3.5 OpenCV and Numpy: Open CV or Open source Computer Vision focuses on real-time computer vision which could be a library of programming functions. Intel was developed this and Willow Garage had supported the OpenCV, and Itseez is preserved it. Under the BSD license of open-source, the library is liberal and is a cross-platform. 
3.5.1 Applications: The application zones of OpenCV involving:

- Mobile AI

- Human-computer Interaction (HCI)

- Gestures recognition

- System for face recognition

- Egomotion estimation

- 2D\&3D feature toolkits

- Understanding of motion

- Objects detection

- Segmentation and recognition A measurable machine learning library is included in Open CV to assist a little of the higher than zones and it includes:

- $\quad$ Support vector machine (SVM)

- Random Forest

- Artificial Neural Networks

- Naïve Bayes Classifier

- K-Nearest Neighbor Algorithm

- Expectation-Maximization algorithm

- Gradient Boosting Trees

- Decision Tree Learning

- Boosting

- Deep Neural Networks (DNN)

The important interface in $\mathrm{C}++$ is included in the OpenCV and composed it conjointly in $\mathrm{C}++$. A broad, and additional seasoned $\mathrm{C}$ interface is there without regarding the holding a less and much reaching. However, the bindings are available in Python, Java, and Matlab/ Octave. Within the on-line documentation, the API is found for these interfaces. To foster the adoption of wrappers extensively, they develop in various languages like Ruby, CH_Haskell, Perl, and C\#. In C++ interface of OpenCv, the bigger a part of the new enhancements and calculations are presently created. NumPy could be considered as a library for the programming language of Python in addition to the support of multi-dimensional and immense matrices, and arrays and accumulating the high-level numerical functions largely for figuring out these arrays. With commitments from a couple of totally different engineers, the ancestor of NumPy, Numeric, was originally made by Jim Hugunin . In 2005, NumPy was created by Travis Oliphant by consolidating highlights of the competitive Numarray into Numeric, with broad changes. NumPy has various benefactors because it is open-source programming.

3.6 Amazon web Services (AWS): Amazon.com has an auxiliary part as Amazon Web Services (AWS) that provides the stages of on-request cloud computing for governments, organizations, and people. Based on a complementary setup selection, it is available to accessible for a year on a paid membership. The PCs with a simple virtual cluster can access perpetually on the online are allowed in the innovation. Virtual computers with AWS's version have played a key role in the real computer qualities together with instrumentality (GPU(s) and CPU(s) for hard-plate stockpiling, RAM memory, and making ready); pre-stacked application programming; organizing; and a call of operating frameworks. For example, databases, web servers, CRM, etc. The facultative AWS subscribers and console I/O (mouse, show, and console) virtualize by every AWS system with the AWS system using a contemporary browser. Here, this browser can display as a window to log into the virtual computer, allow the subscribers to sign up, prepare, and use the virtual systems as a true physical laptop. Based on the administrations for their clients and own advantages, AWS systems will send for granting net initially.

Implementation: This paper is implemented in an effective way by classifying the procedure into two categories. First thing is the working of the hardware used, In this paper the hardware used includes Intel Edison module and an image sensor. The implementation of hardware is described in the Fig.4.1 shown below. The pictures of vehicles are caught by the image sensor and the caught pictures are sent to the edge device to process the image and extract the text from that image. For the processing of the image for extracting the number plate from the image, we use Open CV and Numpy libraries. Processing includes gray scale imaging i.e., image is converted from RGB to gray scale image. Then, through machine learning techniques like KNearest Neighbors algorithm (KNN) we would get to know the number plate of the vehicle and through crop function in Open CV the image is cropped. This is observed in Fig.4.2.Thresholding function is applied, characters are extracted from the threshold image by Optical Character Recognition(OCR). It can be seen in Fig.4.4.In OCR there would be aloha, numeric are predefined and by comparing with these the characters will be assigned. After processing the obtained number plate is checked. If the obtained number is valid then the data is sent to the cloud.

Here the Fig 4.3 describes about the implementation of the user interface. Webpage is utilized as a UI (user interface). (http://13.126.21.59/SmartSpy/V.html). The client enters the required vehicle number in the field given in the webpage. The different fields are likewise accessible with the end goal of confirmation. After submitting the details in the webpage the data already stored will be checked and if the required number is valid the available area of the vehicle will be displayed. (http://13.126.21.59/SmartSpy/data_display.php)

5. Experimental Study: This system uses image sensor to capture the images and Intel Edison to process and send the data to store it in the server. The Fig 5.1 shows the hardware used. Here is the Fig. 5.2 is the webpage to manually enter the culprit number plate. This IP is global IP. The below fields are mentioned for future scope so that there ought not be any misuse. If they want to misuse it, they would be caught because they are providing mobile number to which the verification code i.e., One Time Password is sent. After submission of the vehicle number it gives an acknowledgment that number is entered successfully. Now if cops need to know the vehicle area, it will be visible if it is detected.Area of the vehicle detected will be shown along with date and time. If a vehicle number is invalid or not been detected, at that point it would show as appeared in Fig.5.5. 

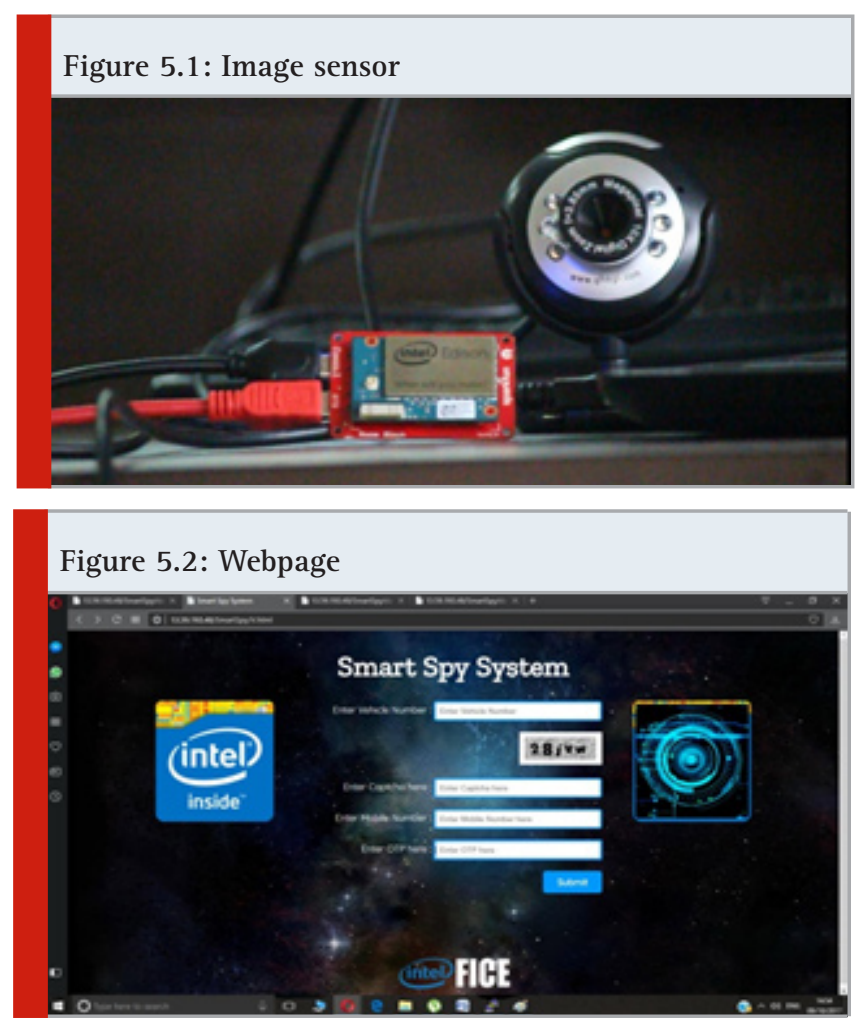

Figure 5.3: Execution process

\section{< > C 88 @ (9) 13.59.193.48/SmartSpy/Nehicleinputphp}

Successfully Entered the Number

\section{CONCLUSION}

We can ensure that crimes can be resolved within less time and the victim can trust that they can get back their valuable things which are lost. It is a financially savvy system which requires less hardware. In future, cops would still find hard time in finding criminals by knowing their vehicle number and location so when we detect number plate of required vehicle it would also detect the face and send it to the cops so that it would be easy enough to get them.
Figure 5.4

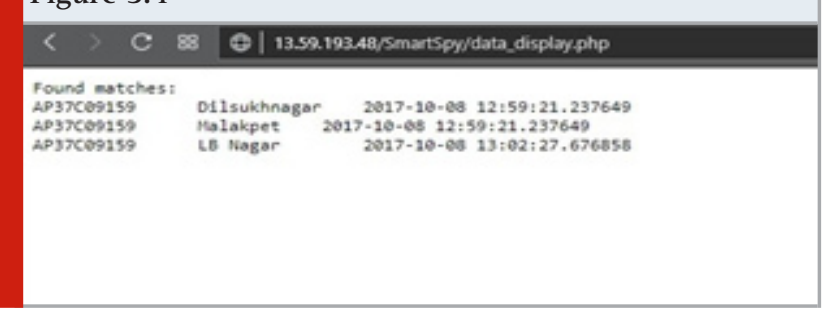

Figure 5.5

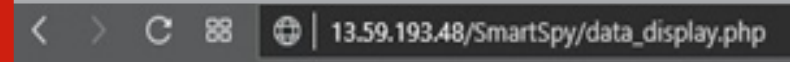

No matches found

\section{REFERENCES}

Anu Agarwal, Sudhir Goswami,( 2016) "An Efficient Algorithm for Automatic Car Plate Detection \& Recognition", Computational Intelligence $A$ Communication Technology (CICT) 2016 Second International Conference on, pp.644-648.

Hatim Derrouz, Azeddine El Hassouny, RachidOulad Haj Thami, Hamid Tairi,( 2017) "Hybrid method for background modeling and subtracting", Intelligent Systems and Computer Vision (ISCV) 2017, pp. 1-5. Nandan More, Bharat Tidke, "License Plate Identification Using Artificial Neural Network and Wavelet Transformed Feature Selection", Pervasive Computing (ICPC) 2015 International Conference on, pp. 1-5, 2015.

Intel Edison and sparkfunbaseblock manual [online] Available:https://learn.sparkfun.com/tutorials/generalguide-to-sparkfun-blocks-for-intel-edison

Nauman Saleem, Hassam Muazzam, H. M. Tahir, Umar Farooq, (2016) "Automatic license plate recognition using extracted features", Computational and Business Intelligence (ISCBI) 2016 4th International Symposium on, pp. 221-225.

Open CV and python tutorials [online] Available:https:// docs.opencv.org/3.0-beta/doc/py_tutorials/py_setup/ py_intro/py_intro.html.

Y. Y. Nguwi, W. J. Lim, "Number plate recognition in noisy image", Image and Signal Processing (CISP) 2015 8th International Congress on, pp. 476-480, 2015. 